\section{Expression of TetC \\ Fusion Proteins from \\ Salmonella in a Gaseous \\ Environment that Models \\ Conditions Found in \\ Mammalian Tissues}

BioTechniques 28:228-232 (February 2000)

There is an increasing body of evidence to suggest that attenuated strains of Salmonella can be used as live multivalent vaccine delivery systems for guest antigens from other pathogens. However, the constitutive expression of guest antigens, particularly from eukaryotic pathogens, can result in segregation and loss of the recombinant expression plasmid. To maintain expression of these guest antigens, an in vivo inducible promoter can be used. This offers an attractive alternative to the use of plasmids containing genes that confer antibiotic resistance, which is routinely used in vitro but is not acceptable for clinical applications. The modified Escherichia coli nitrite reductase nirB promoter is induced under conditions of reduced oxygen tension and has been used with great success to direct the expression of guest antigens in vivo (8). An attenuated S. typhi construct harboring a plasmid containing fragment-C from tetanus toxin (TetC) of Clostridium tetani under the transcriptional control of nirB is under evaluation as a combined human typhoid-tetanus vaccine (5). The Salmonella-TetC expression system has been used to enable the expression of a variety of antigens fused to TetC.

The induction of the nir $\mathrm{B}$ promoter has been described using a number of different industrial anaerobic induction regimes. These regimes have proved impractical for scaling down to suit primary research purposes. This report compares expression of antigens from the human malaria parasite Plasmodium falciparum with TetC as a fusion partner driven from the nirB promoter in aerobic, microaerophilic and anaerobic conditions, using a commercially available small-scale atmosphere generating system. We show that the nirB promoter is inducible under microaerophilic conditions that model those found in lymphoid organs colonized by Salmonella in vivo and so can be used as a tool to predict the efficacy of a multivalent vaccine before administration.

The malarial antigens studied were the $\mathrm{C}$ - and N-terminal non-repeat regions of liver stage antigen-1 (LSA-1) (11). Immune responses to peptides within the $\mathrm{N}$-terminal region are associated with an absence of blood stage parasitemia (1) and with protection against severe malaria (4). The codon bias of a gene can have a major impact on the expression level of the corresponding protein and hence its immunogenicity in a live bacterial vaccine. Increased expression of TetC after codon optimization may have contributed to the success of the Salmonella-TetC tetanus vaccine (5). The gene fragments of the malarial antigens used in this study were not codon-optimized for bacterial expression. The use of $\mathrm{A}+\mathrm{T}$ bases in the genome of $P$. falciparum, at greater than $80 \%$, is higher than any other organism whose DNA has been characterized (9). This codon bias is a novel feature of the genome of this protozoan parasite and differs significantly from Salmonella, which has an $\mathrm{A}+\mathrm{T}$ content of $48 \%$. Hence, it may be predicted that malarial antigens may be expressed at low levels in a Salmonella host.

Vaccine formulations of Salmonella are grown aerobically to a stationary phase. When administered orally, the bacteria persist in the lymphoid organs, such as liver and spleen, until cleared by the host's immune system (5). To further our research on the use of Salmonella as a vaccine delivery system for malaria antigens, we sought to determine the expression of TetC fusion proteins from a vaccine strain. Previous studies have shown that expression of the lac $Z$ reporter gene from nirB increases significantly when Salmonella enter mammalian macrophages in vitro (2). As direct examination of fusion protein expression in vivo is not feasible, we have developed a method by which expression can be determined

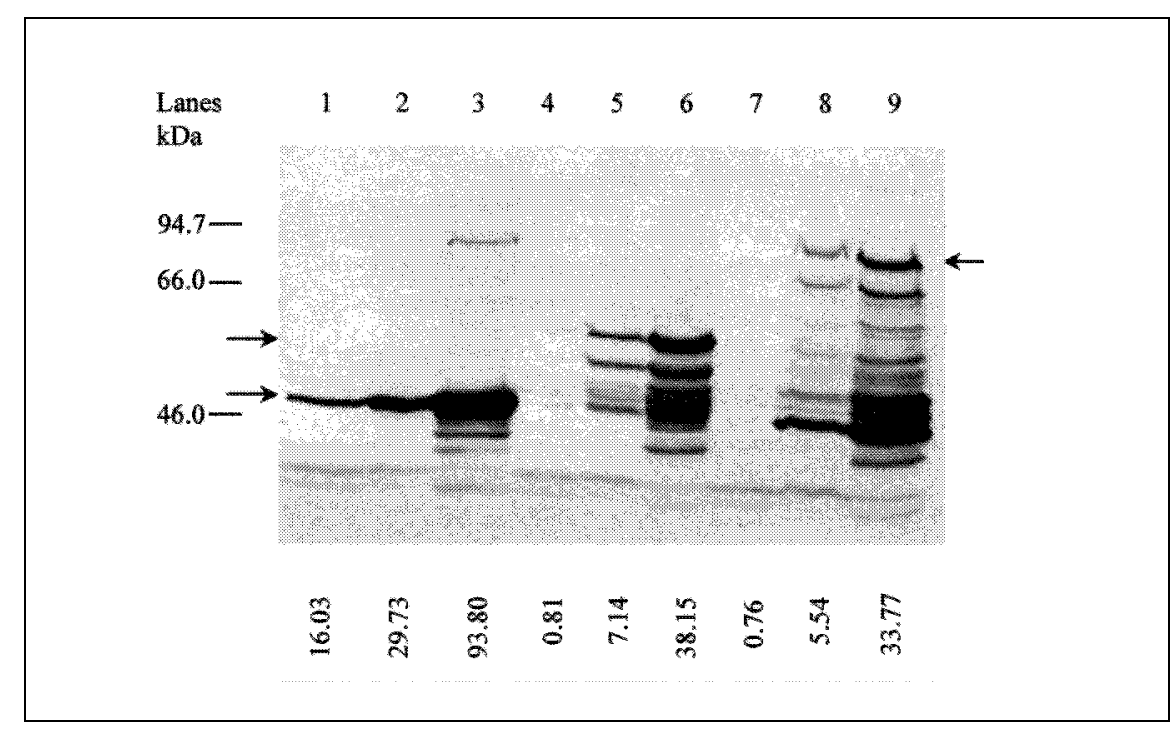

Figure 1. Comparison of expression levels of TetC and TetC fusion proteins by $S$. typhimurium when grown under conditions of varying oxygen tension, analyzed by Western blotting using antiTetC polyclonal serum. Expression of the N-terminal and C-terminal non-repeat regions of $P$. falciparum LSA-1 as TetC fusion proteins by $S$. typhimurium strain BRD726 was compared to that of TetC under aerobic, microaerophilic and anaerobic conditions. TetC, lanes 1-3; N-terminal region TetC fusion protein, lanes 4-6; C-terminal region TetC fusion protein, lanes 7-9. Aerobic, lanes 1, 4 and 7; microaerophilic, lanes 2, 5 and 8; anaerobic, lanes 3,6 and 9. Arrows indicate the positions of full-length $\mathrm{TetC}$ and TetC fusion proteins. Band intensities, measured as adjusted volume (Molecular Analysis software; Bio-Rad Laboratories, Hemel Hempstead, England, UK), are shown under each lane. The bands representing proteins of lower molecular weight are those that contain the full-length TetC but truncated LSA-1 regions. Expression of TetC fusion proteins under aerobic conditions cannot be seen (lanes 4 and 7) and was only detectable when the highly sensitive detection method of enhanced chemiluminescence was used (data not shown). The $93 \mathrm{kDa}$ band seen in lane 3 is a stress protein synthesized when TetC is overexpressed. Similar results were obtained for three other attenuated strains of S. typhimurium. 
from Salmonella grown in gaseous environments that simulate those found in mammalian tissues. Oxygen levels produced by the microaerophilic atmosphere generated by CampyGen ${ }^{\mathrm{TM}}$ gas sachets (Oxoid, Basingstoke, England, UK) are of a similar range, $4 \%-8 \% \mathrm{O}_{2}$, to those that occur in extracellular locations in vivo (3). In support of this simulation of physiological conditions, we found that the use of CampyGen gas sachets sustains the growth of blood stage cultures of $P$. falciparum (10). This method of incubation provides a simple, inexpensive and rapid means by which to assess the expression of the TetC malaria fusion proteins and has generated valuable data used to evaluate which constructs to use for immunization.

In these experiments, stationary phase bacteria were either seeded onto LB agar or inoculated into LB broth. The cultures were then placed into an airtight gas jar (Anaerojar ${ }^{\mathrm{TM}}$; Oxoid) into which either an AnaeroGen ${ }^{\mathrm{TM}}$ or CampyGen gas generating sachet was added. The sachets were activated following the manufacturer's instructions to generate anaerobic $\left(<1 \% \mathrm{O}_{2}, 9 \%\right.$ $\left.\mathrm{CO}_{2}, 90 \% \mathrm{~N}_{2}\right)$ and microaerophilic (6\% $\mathrm{O}_{2}, 8 \% \mathrm{CO}_{2}, 86 \% \mathrm{~N}_{2}$ ) conditions, respectively. Bacteria were harvested in LB broth by centrifugation, washed in PBS and the amount of bacteria per sample was adjusted to an equivalent level based on $\mathrm{A}_{650}$ values. Levels of expression of the TetC fusion proteins were determined by SDS-PAGE and Western blotting using polyclonal serum against TetC or specific malarial antigens (7).

Figure 1 shows the levels of expression of the TetC-LSA-1 fusion proteins increased with decreasing oxygen tension for a representative vaccine strain of S. typhimurium. C5htrA (BRD726) (5). This inverse correlation was also shown for each of three other different attenuated strains of S. typhimurium examined (C5htrA, SL3261 aroA, C5aroD) (data not shown), indicating the probability of high level expression under the microaerophilic conditions that prevail within tissues in vivo. It also provides a formal demonstration that the nirB promoter has an anaerobic optimum. To our knowledge, this is the first time expression of guest antigens from Salmonella not codon-optimized for bacterial expression has been shown under the tran- scriptional control of the nir B promoter.

Expression of the TetC-LSA-1 fusion proteins under aerobic conditions was weak compared to that of TetC alone (ca. 95\% less protein; Figure 1 lanes 1, 4 and 7). Expression of heterologous recombinant proteins may be limited when the codon usage of the recombinant gene differs from that of the host cell. Induced expression of mRNA encoding proteins with rarely used codons often leads to inefficient translation of the mRNA. This can result in the premature termination of translation and/or poorly expressed levels of the full-length protein. (6). An investigation of the inducibility of the TetC-LSA-1 fusion proteins was important to decide whether immunizations should proceed.

Our past experience of this vaccine delivery system suggested that significantly increased expression of these antigens in vivo would be required to elicit an immune response. There is currently no method available to determine expression levels of fusion proteins in vivo, although rescue of bacteria, and thus of expression plasmids, may be achieved from host organs to enable an evaluation of plasmid stability. The method described here provides a novel way to determine maximal expression of fusion proteins under microaerophilic conditions similar to those found in host lymphoid organs where invasion by Salmonella takes place. This provides a useful tool for predicting the in vivo expression levels of a particular guest antigen and for selecting the optimal attenuated vaccine host strain before administration.

The application of the commercial gas generation system to produce anaerobic and microaerophilic atmospheres enabled these experiments to proceed on a small scale and thus produced reproducible data without expert knowledge of large-scale fermenters. The technique may be adapted for growth of other facultatively anaerobic or microaerophilic pathogens for which fusion protein expression from a promoter may be maximally induced under conditions of reduced oxygen tension. In addition to Salmonella, examples of these include the bacteria Listeria, Mycobacterium, Vibrio and Yersinia, and the intracellular protozoa Plasmodium, Leishmania, Toxoplasma and Trypanosoma. 


\section{REFERENCES}

1.Connelly, M., C.L. King, K. Bucci, S. Walters, B. Genton, M.P. Alpers, M. Hollingdale and J.W. Kazura. 1997. T-cell immunity to peptide epitopes of liver-stage antigen 1 in an area of Papua New Guinea in which malaria is holoendemic. Infect. Immun. 65:5082-5087.

2.Everest, P., G. Frankel, J. Li, P. Lund, S. Chatfield and G. Dougan. 1995. Expression of LacZ from the htrA, nirB and groE promoters in a Salmonella vaccine strain: influence of growth in mammalian cells. FEMS Microbiol. Lett. 126:97-101.

3.Harris, E.A., A.M. Kenyon, H.D. Nisbet, E.R. Seelye and R.M. Whitlock. 1974. The normal alveolar-arterial oxygen-tension gradient in man. Clin. Sci. Mol. Med. 46:89-104.

4.Hill, A.V.S., J. Elvin, A.C. Willis, M. Aidoo, C.E.M. Allsopp, F.M. Gotch, X.M. Gao, M. Takiguchi et al. 1992. Molecular analysis of the association of HLA-B53 and resistance to severe malaria. Nature 360:434-439.

5.Hormaeche, C.E. and C.M.A. Khan. 1996. Recombinant bacteria as vaccine carriers of heterologous antigens, p. 327-359. In S.H.E. Kaufmann (Ed.), Concepts in Vaccine Development. Walter de Gruyter, Berlin.

6.Kane, J.F. 1995. Effects of rare codon clusters on high-level expression of heterologous proteins in Escherichia coli. Curr. Opin. Biotechnol. 6:494-500.

7.Khan, C.M.A., B. Villarreal-Ramos, R.J. Pierce, G. Riveau, R. Demarco de Hormaeche, H. McNeill, T. Ali, S. Chatfield et al. 1994. Construction, expression, and immunogenicity of the Schistosoma mansoni P28 glutathione S-transferase as a genetic fusion to tetanus toxin fragment $\mathrm{C}$ in a live Aro attenuated vaccine strain of Salmonella. Proc. Natl. Acad. Sci. USA 91:11261-11265.

8.Oxer, M.D., C.M. Bentley, J.G. Doyle, T.C. Peakman, I.G. Charles and A.J. Makoff. 1991. High level heterologous expression in $E$. coli using the anaerobically-activated nirB promoter. Nucleic Acids Res. 19:2889-2892.

9.Pollack, Y., A.L. Katzen, D.T. Spira and J. Golenser. 1982. The genome of Plasmodium falciparum. I: DNA base composition. Nucleic Acids Res. 10:539-546.

10.Taylor-Robinson, A.W. 1998. Cultivation of Plasmodium falciparum in vitro using microbiological gas sachets that generate microaerophilic conditions. Trans. Roy. Soc. Trop. Med. Hyg. 92:357-358.

11.Zhu, J. and M.R. Hollingdale. 1991. Structure of Plasmodium falciparum liver stage antigen-1. Mol. Biochem. Parasitol. 48:223-226.

The authors wish to thank Dr. Hesta McNeill for helpful discussions. This work was supported by the Wellcome Trust. A.W.T.-R. is a Wellcome Trust Research Career Development Fellow in Basic Biomedical Science. Address correspondence to Dr. Andrew Taylor-Robinson, School of Biology, University of Leeds, Clarendon Way, Leeds LS2 9JT England, UK. Internet: bgyawtr@ leeds.ac.uk
Received 18 August 1999; accepted 27 October 1999.

K.G. Heal, G.A. McConkey, C.E. Hormaeche ${ }^{1}$, M.R. Hollingdale, C.M.A. Khan ${ }^{1}$ and A.W. Taylor-Robinson University of Leeds, Leeds ${ }^{1}$ University of Newcastle Newcastle, England, UK

\section{Recovery of Protein Kinases from Renatured SDS-Polyacrylamide Gels for Biochemical Studies}

BioTechniques 28:232-238 (February 2000)

The in-gel kinase assay (8) has been widely used to detect novel protein kinases in different types of cells. Using different peptides, such as myelin basic protein (MBP), bacteria-produced cJun peptide and polydispersed co-polymer of glutamic acid and tyrosine [poly(Glu, Tyr)] as substrates immobilized in SDS-polyacrylamide gel (SDSPAG), cellular proteins are electrophoresed. The kinase activity and molecular masses of many protein kinases were measured and detected, including MAPK/Erk (mitogen-activated protein kinase/extracellular signal regulated kinase) $(9,16)$, SAMK/Krs1/2 (src-activated MBP kinase/kinases responsive to stress) $(15,16)$, JNK/SAPK (Jun N-terminus kinase/stress activated protein kinase) (3) and several novel protein tyrosine kinases $(6,7,10)$.

The advantages of the in-gel kinase assay are its sensitivity and broad application for detection of different protein kinases in crude cell lysates containing as little as $5 \mu \mathrm{g}$ of total cellular proteins (11) in a substrate-specific manner. One limit of this technique is its inability to detect kinases that may not be renatured in SDS-PAG. Here, we report an extended application of the in-gel kinase assay to the biochemical study of protein kinases. After renaturation, pro- tein kinases were recovered from SDSPAG slices to determine their kinase activities and detect phosphoamino-acid in substrate phosphorylated by the kinase. Use of this new protocol for the study of a newly detected protein kinase in a crudely prepared cell lysate provides information that usually requires the purified protein kinase.

Cultures of primary chicken embryo fibroblast (CEF) cells were prepared from $g s$-negative embryos (SPAFAS, Norwich, CT, USA) grown in DMEM (Life Technologies, Gaithersburg, MD, USA) that were supplemented with $1 \%$ chicken serum, $4 \%$ calf serum, $50 \mathrm{U} /$ $\mathrm{mL}$ penicillin, $5 \mathrm{mcg} / \mathrm{mL}$ streptomycin, $10 \%$ tryptose phosphate broth (2) and incubated at $37^{\circ} \mathrm{C}$. Cells were routinely subcultured every 2 or 3 days. Cells were transfected with pRL-src, an infectious, nonpermuted molecular clone of PrA RSV, a process that results in viral production and transmission within the cell culture (13). Typically, $1 \mu \mathrm{g}$ of pRL DNA was used to transfect one 60 mm dish of chicken embryo cells $24 \mathrm{~h}$ following trypsinization and plating. Changes in cell morphology were routinely observed 6-8 days following transfection. To prepare cytosolic proteins, cultures were rinsed with ice-cold PBS, and the cells were scraped from culture dishes. They were then lysed with 30 strokes of a tight-fitting Dounce homogenizer in lysis A buffer (10 $\mathrm{mmol} / \mathrm{L} \mathrm{KH}_{2} \mathrm{PO}_{4}, 1 \mathrm{mmol} / \mathrm{L}$ EDTA, 5 $\mathrm{mmol} / \mathrm{L}$ EGTA, $10 \mathrm{mmol} / \mathrm{L} \mathrm{MgCl}_{2}, 50$ $\mathrm{mmol} / \mathrm{L} \beta$-glycerol phosphate, $1 \mathrm{mmol} /$ $\mathrm{L} \mathrm{Na}_{3} \mathrm{VO}_{4}, 2 \mathrm{mmol} / \mathrm{L}$ dithiothreitol [DTT]) supplemented with $1 \mathrm{mmol} / \mathrm{L}$ phenylmethylsulfonyl fluoride, 4 $\mathrm{mmol} / \mathrm{L}$ p-nitrophenyl phosphate disodium and $1 \mathrm{mmol} / \mathrm{L}$ pepstatin. Crude lysates were centrifuged at $150000 \times g$ for $30 \mathrm{~min}$ at $4^{\circ} \mathrm{C}$ to separate cytoplasmic supernatant from crude membrane and nuclei pellets (16). Protein concentration in cellular lysates was measured using the BCA assay (Pierce Chemical, Rockford, IL, USA).

To detect protein kinases in cell lysates, $20 \mu \mathrm{g}$ of cellular proteins isolated from normal and $s r c$-transformed CEF were resolved in two separate SDS-PAGs in the absence (Figure 1A) or presence (Figure 1B) of $0.4 \mathrm{mg} / \mathrm{mL}$ of MBP (Life Technologies) as a substrate immobilized in the gel. After elec- 